Édition préparée par

Maria Francesca Buonaiuto (texte), Marina Pierobon (illustrations)

Composition de la couverture par Marina Pierobon

Mise en page par Véronique Cassou 


\section{IMAGE ET RELIGION}

\section{DANS L'ANTIQUITÉ GRÉCO-ROMAINE}


Image et religion dans l'antiquité gréco-romaine : actes du colloque de Rome, 11-13
décembre 2003, organisé par l'École française de Rome, l'École française d'Athènes,
l'ArScAn (UMR 7041: CNRS, Paris I, Paris X), l'équipe ESPRI et l'ACI jeunes chercheurs
ICAR / publiés sous la dir. de S. Estienne, D. Jaillard, N. Lubtchansky et Cl. Pouzadoux ;
- Naples : Centre Jean Bérard ; École française d'Athènes, 2008. - 502 p. : ill. ; 30 cm.
(Collection du Centre Jean Bérard, ISSN 1590-3869 ; 28)
ISBN 978-2-903189-95-2 (Centre Jean Bérard)
ISBN 978-2-86958-236-1 (École française d'Athènes)
I. Estienne, Sylvia. II. Jaillard, Dominique. III. Lubtchansky, Natacha. IV. Pouzadoux,
Claude. V. Centre Jean Bérard. VI. École française de Rome. VII. École française
d'Athènes. VIII. ArScAn (UMR 7041: CNRS, Paris I, Paris X). IX. Équipe ESPRI. X. ACI
jeunes chercheurs ICAR.
CIP - Centre Jean Bérard

Diffusion De Boccard 11, rue de Médicis 75006 Paris
L'ERMA di Bretschneider

Via Cassiodoro, 19

00193 Roma
M. D’Auria Editore

Calata Trinità Maggiore,52

80134 Napoli
EDIPUGLIA

Via Dalmazia, 22/B

70050 Bari - S. Spirito 
Collection du Centre Jean Bérard, 28

\section{IMAGE ET RELIGION \\ DANS L'ANTIQUITÉ GRÉCO-ROMAINE}

Actes du Colloque de Rome

11-13 décembre 2003

organisé par l'École française de Rome,

l'École française d'Athènes,

l'ArScAn (UMR 7041: CNRS, Paris I, Paris X),

l'équipe ESPRI et l'ACI jeunes chercheurs ICAR

publiés

sous la direction de

S. Estienne, D. Jaillard, N. Lubtchansky et Cl. Pouzadoux

par le Centre Jean Bérard, l'École française de Rome, l'École française d'Athènes avec le concours de l'Université de Paris X - Nanterre et le soutien financier d'ArScAn, de l'équipe ESPRI et de l'École doctorale "Milieux, cultures et sociétés du passé et du présent » 


\title{
Des marmites pour un méchant petit hermès ! ou comment consacrer une statue
}

\author{
Vinciane Pirenne-Delforge*
}

\section{Introduction}

L'interjection choisie comme première partie du titre de cet article est la citation du vers 924 de la comédie d'Aristophane intitulée la Paix, la déesse délivrée par Trygée afin de contrer les méfaits du dieu Polémos. Trygée propose donc à son serviteur "d'installer la déesse par des marmites ». Et le serviteur de rétorquer brusquement : "Par des marmites ? Comme un méchant petit hermès ?" Il désigne ainsi un petit pilier hermaïque et tout dans ses propos laisse entendre qu'une telle " installation » est indigne de la précieuse déesse délivrée à grand peine ${ }^{1}$. Les compères changent alors de stratégie et envisagent successivement un bœuf gras, un cochon et un mouton sur lequel se portent finalement leurs suffrages ${ }^{2}$. La scène du sacrifice en l'honneur de la Paix se déploie juste après, et avec elle l'un des rares témoignages un peu circonstanciés sur les différentes phases de la procédure sacrificielle en Grèce ${ }^{3}$. Le Ploutos, cette autre comédie d'Aristophane, offre une scène parallèle où il s'agit de réinstaller la Richesse divinisée dans l'opisthodome d'Athéna qu'elle n'aurait jamais dû quitter : une sorte de procession mène le dieu, entouré de porte-torches et d'une vieille qui porte sur la tête les marmites par lesquelles le dieu sera installé ${ }^{4}$. L'expression est chaque fois identique :

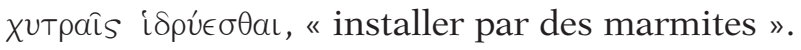

Les scholies à ces deux passages stipulent qu'on pouvait installer un dieu en faisant bouillir des céréales dans des marmites ou en choisissant une victime plus coûteuse. D'où l'expression complémentaire : "installer par un bœuf, par une chèvre ou par du menu bétail ». Comme souvent dans un tel cas, les différents scholiastes et lexicographes plus ou moins tardifs se sont allègrement recopiés et fournissent, au départ de ces quelques vers, des lectures redondantes, avec de faibles variations. En l'occurrence, la Souda offre une glose plus développée que les autres ${ }^{5}$. Elle précise qu'il s'agit de

* FNRS - Université de Liège.

Ce travail a bénéficié de discussions fructueuses avec Dominique Jaillard et Francis Prost. Qu'ils en soient remerciés.

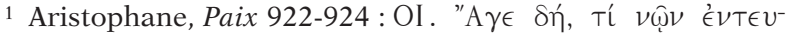

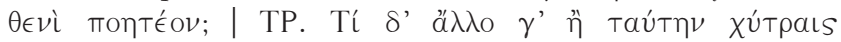

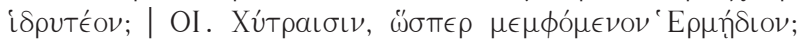

2 Ibid. 926-938.

3 Ibid. 937-1139.

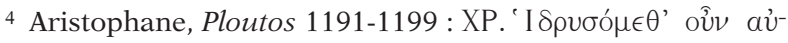

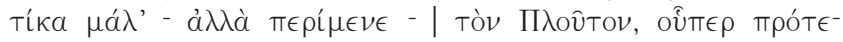

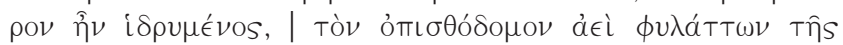

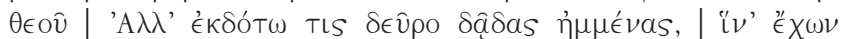

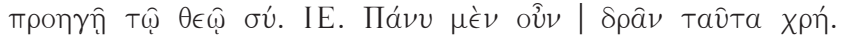

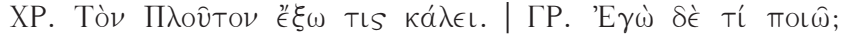

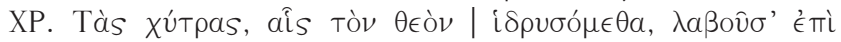

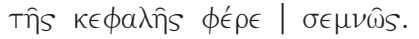

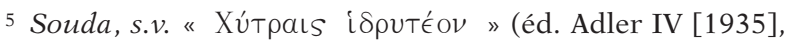

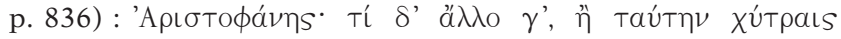

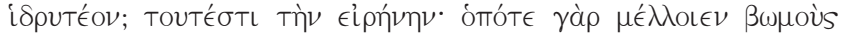

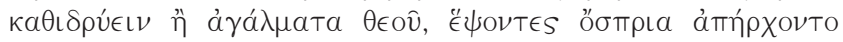

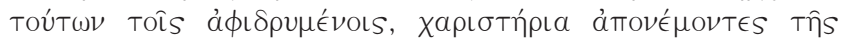

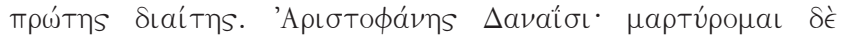

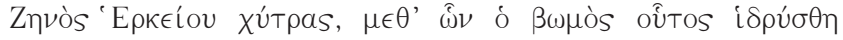

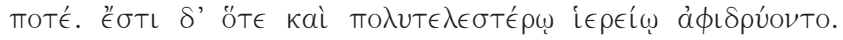

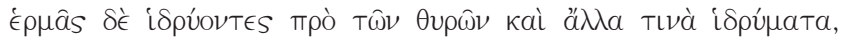

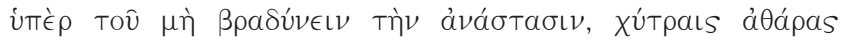

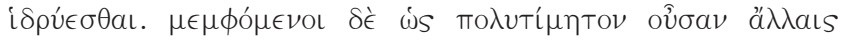

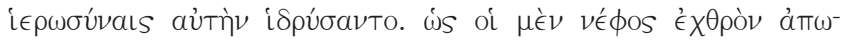

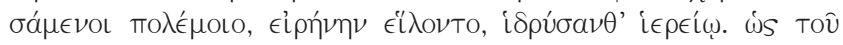

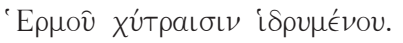


l'installation de statues et d'autels. La cuisson d'une bouillie de légumineuses aurait pour but de commémorer le premier régime alimentaire. On y apprend encore qu'une comédie perdue d'Aristophane mettait en scène un personnage prenant à témoin " les marmites de l'Herkeios [c'est-à-dire Zeus] avec lesquelles cet autel avait été installé » [c'est-à-dire celui près duquel il jurait]. Il était bien entendu possible d'offrir des victimes de plus de prix, mais pour aller vite et ne pas retarder l'élévation des piliers devant les portes et d'autres installations du même genre, on utilisait des marmites de bouillie. L' « installation par des marmites » est donc une pratique dont les gloses attestent le caractère modeste et rapide, d'une part, mais aussi l'équivalence structurelle avec une immolation animale. Elles placent en outre sur un pied d'égalité les procédures mises en œuvre pour les statues et pour les autels, ce qui est particulièrement intéressant dans le cadre de la problématique envisagée ici.

Ce questionnement est né d'une réflexion du regretté Jean Rudhardt. En effet, dans l'article des MEFRA qui publie sa participation au premier séminaire Image et religion ${ }^{6}$, on peut lire (p. 184) : "À l'exception peut-être de quelques statues miraculeuses... toutes les statues divines sont dites des anathèmata. À ce titre elle firent l'objet d'une opération rituelle. " L'auteur ne s'explique pas davantage sur cette affirmation, mais tout le contexte de sa réflexion implique l'idée d'une "consécration". Pour Simona Bettinetti, dans un ouvrage récent intitulé La statua di culto nella pratica rituale greca, une statue de culte peut se définir de deux points de vue : en référence à l'éventuelle dimension surnaturelle et donc miraculeuse de l'objet, ou à son rapport à un culte ou à un rituel déterminé 7 . Fritz Graf écrivait, récemment lui aussi, que la Grèce, contrairement à Rome ou au Proche-Orient, ne connaissait pas de rituel de consécration qui serait venu fonder le statut particulier, divin, de l'image ${ }^{8}$. Quant à Alice Donohue, elle ne pose pas le problème d'une "consécration", mais relègue purement et simplement la notion même de "statue de culte" au magasin des accessoires conceptuels hérités d'une certaine "anticomanie" et des polémiques iconoclastes $^{9}$.

Une telle absence de convergence dans des travaux récents et sous la plume de bons connaisseurs du système religieux grec invite à formuler deux remarques sous forme de deux questions étroitement solidaires :
1 - dans quelle mesure peut-on assimiler "statue divine" et "statue de culte" ? 10

2 - la notion un peu vague de "consécration" d'une statue est-elle pertinente pour la Grèce et, dans l'affirmative, à quel contenu et à quelle signification "grecs" peut-on l'associer ? 11

Le petit dossier des gloses sur les marmites d'Aristophane est le seul élément vraiment tangible et circonstancié de la documentation littéraire ${ }^{12}$.

6 J. Rudhardt, La perception grecque du territoire sacré, MEFRA, 113, 2001, p. 175-188.

7 Bettinetti 2001, p. 7.

8 Graf 2001, citation p. 230 : « ... anders als in Rom, im Alten Orient und in der christilichen Spätantike fassen wir für griechische Götterbilder keine rituellen Weihungen, welche einen besonderen, göttlichen Status des Bilds begründet hätten. » Voir aussi Linant de Bellefonds 2004, p. 418, et les deux importants articles de B. Gladigow : Präsenz der Bilder - Präsenz der Götter. Kultbilder und Bilder der Götter in der griechischen Religion, Visible Religion, 4-5, 1985-1986, p. 114-133, spéc. p. 118 ; Epiphanie, Statuette, Kultbild. Griechische Gottesvorstellungen im Wechsel von Kontext und Medium, Visible Religion, 7, 1990, p. $98-121$.

9 A.A. Donohue, The Greek Images of the Gods. Considerations on terminology and methodology, Hephaistos, 15, 1997, p. 31-45.

10 Linant de Bellefonds 2004 tranche le problème de l'extension du chapitre "Images de culte » de ce Thesaurus en choisissant de traiter de toutes les catégories d'images divines, sans isoler les statues dressées à un emplacement privilégié du sanctuaire - ce qui semble donc être pour elle le critère de « cultuelle».

11 Sur la "consécration” en Grèce, il n'existait jusqu'il y a peu qu'un seul ouvrage assez ancien: G. Hock, Griechische Weihegebraüche, Würzburg, 1905. On dispose aujourd'hui d'un copieux chapitre du ThesCRA sur le sujet: V. Lambrinoudakis et al., Consecration, Foundation Rites, in : ThesCRA III, Los Angeles, 2005, p. 303-346. Malheureusement, l'article n'intègre pas de discussion réelle de la consécration des objets de culte, simplement reprise sous le titre de «Dedication of cult objects » où le lecteur est renvoyé aux autres chapitres : Altars, Cult Instruments, Offerings, Statues. - Je dissocie ici la "consécration" des temples (cf. BCH, 71-72, 1947/48, p. 148-254, spéc. 248-254) de celle des autels et statues.

12 Pour l'utilisation de marmites dans le cadre de certaines offrandes aux morts, $c f$. les analyses de X. De Schutter, La marmite et la panspermie des morts, Kernos, 9, 1996, p. 333-345. $\mathrm{Cl}$. Rolley interprétait, quant à lui, la procession du Ploutos comme une parodie de la kernophorie éleusinienne et limitait l'offrande de marmites aux daimones dispensateurs de richesses : Revue des Études Grecques, 79, 1966, p. XIV-XV. 
Quant à la documentation épigraphique, elle est, elle aussi, désespérément allusive ${ }^{13}$. Dès lors, en résonance avec les précieuses recherches de Jean Rudhardt sur les notions fondamentales ${ }^{14}$, voyons quels sont les verbes utilisés dès qu'il s'agit de statues divines.

\section{Des questions de vocabulaire}

"Consacrer" signifie qu'un objet ou un être entre dans la propriété des dieux. La dédicace fait partie de telles procédures, sous l'étiquette familière des anathèmata. L' anathèma est au sens strict une déposition en faveur d'un dieu ${ }^{15}$. Quoi qu'écrive J. Rudhardt dans l'extrait mentionné ci-dessus, nous n'avons pas de trace d'une opération rituelle particulière au-delà de la simple déposition. C'est le contexte qui "sacralise" l'objet et le soustrait à tout autre usage. Seule une manipulation ultérieure des objets en question requiert quelque précaution. Ainsi, la refonte d'ex-voto implique de conserver soigneusement la liste des anciens dédicants et d'assortir éventuellement l'opération d'un sacrifice propitiatoire. Le sacrifice vise à éviter la colère du dieu devant la manipulation de ses avoirs, mais n'entre pas dans une quelconque "sacralisation" du nouvel anathèma ${ }^{16}$. Une inscription de Rhodes montre que la dédicace d'un particulier impliquait l'introduction d'une demande auprès des responsables du sanctuaire qui, dans le cas d'un Asclépieion notamment, devaient contrôler sévèrement la déposition ${ }^{17}$. Mais un tel contrôle semble avoir eu pour objectif principal d'éviter les accumulations en des lieux inappropriés et non de soumettre l'objet en question à une quelconque opération "sacralisante" 18. Cette inscription mentionne en outre clairement les éléments que vise la réglementation : il s'agit des andriantes et des " autres anathèmata ${ }^{19}$.

Un tel passage dans la sphère de la propriété divine, s'il est surtout marqué par le verbe ảvatín $\mu$, peut aussi être désigné par le verbe, plus explicite sans doute, $\kappa a \theta t \in \rho o ́ \omega$, c'est-à-dire textuellement, rendre hieros, rendre "sacré ». Si l'utilisation du verbe ne souffre pas la moindre comparaison avec la famille des anathèmata, surtout avant la fin de la période hellénistique, ses emplois marquent l'aboutissement d'une action dont anatithèmi souligne les aspects concrets ${ }^{20}$.

Et la statue divine dans tout cela ? Les andriantes de l'inscription de Rhodes ne relèvent pas de cette catégorie. Il faut se tourner vers d'autres termes comme agalma, xoanon, ou plus anciennement, bretas. Les enquêtes terminologiques autour de la statue sont nombreuses ${ }^{21}$ et il ne s'agit pas de reprendre ici ce volumineux dossier. Par contre, l'installation de cette statue est essentielle pour la réflexion présente. Or, si la famille d'anatithèmi ou le verbe kathieroô peuvent apparaître dans un tel contexte, c'est la famille du verbe hidruesthai qui revient de façon récurrente. Nous l'avons vu à propos du dossier que, pour faire bref, j'appellerai "des marmites". Apparenté à l'étymologie des mots de la famille de hezô, hedos, etc., il met l'accent sur la fondation, l'installation, l'établissement, mais dans un registre dont Eustathe pourra dire qu'il est devenu majoritairement "sacré » chez les auteurs attiques $^{22}$. Ce sont des temples, des statues et des autels qui constituent les compléments privilégiés du verbe.

13 Bettinetti 2001, p. 60, cite une inscription de Cos (1 $1^{\text {ere }}$ moitié III ${ }^{\mathrm{e}}$ s. av. J.-C. = LSCG 154 B, l. 11-16), tout en soulignant que les éléments déterminants relèvent de restitutions dues à R. Herzog. Ces restitutions sont tellement importantes qu'il semble vain de verser une telle pièce au "dossier" des consécrations.

14 J. Rudhardt, Notions fondamentales et actes constitutifs $d u$ culte dans la Grèce classique, Paris, 1992², [1958].

15 Ibid., p. 214-218.

16 LSCG 41, 1. 45-47 (221/20 av. J.-C., sanctuaire du Héros

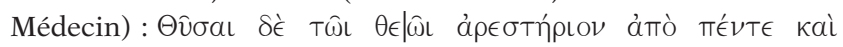

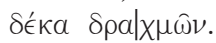

17 LSS 107 (III ${ }^{\mathrm{e}}$ s. av. J.-C.).

18 Cf. aussi LSCG $70=$ B.C. Petrakos, Oi é $\pi \iota \gamma \rho \alpha \phi \dot{s}$ Tôे

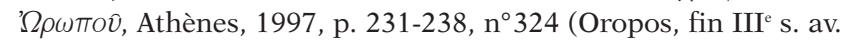
J.-C.),

19 LSS 107, 1. 4-5.

20 Rudhardt, op. cit. (note 14), p. 223-224.

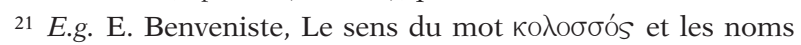
grecs de la statue, Revue de Philologie, 6, 1932, p. 118-135; Vernant 1996, p. 359-377 [reprise d'un article publié en 1983]; A.A. Donohue, Xoana and the Origins of Greek Sculpture, Atlanta, 1988 (American Classical Studies, 15); Scheer 2000, p. 3-34; Bettinetti 2001, p. 25-105.

22 Eustathe, Commentaire à l'Iliade III, 78, 387, 8-11 (éd. Van

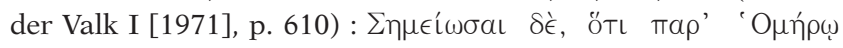

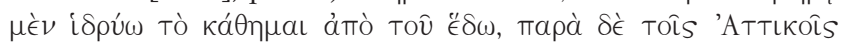

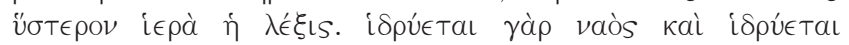

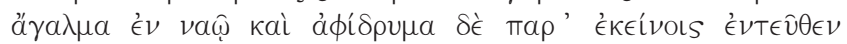

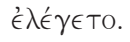


Nous disposons là d'un indice permettant d'introduire une première hypothèse sur l'éventuelle différence entre "statue divine" et "statue de culte". La "statue de culte", même si aucun terme en grec ne correspond exactement à l'expression, est une statue divine qui a été "installée". J'en donnerai deux exemples, tirés l'un d'une tragédie, l'autre d'une inscription. Dans l'Iphigénie en Tauride, Euripide met en scène Oreste poussé par Apollon à rechercher l'antique image d'Artémis. Il explique l'oracle de Loxias à celle qui s'avère être sa sœur Iphigénie : "Enfin, prophétisant du haut du trépied d'or, le dieu m'envoya prendre ici cette statue tombée du ciel, que je devais dresser ensuite en terre athénienne ${ }^{23}$. La statue fait partie des objets "miraculeux", mais l'injonction du dieu vise à lui donner un ancrage déterminé et donc, me semble-t-il, à la transformer en "statue de culte". D'autre part, dans une inscription de Pergame datée du $\mathrm{II}^{\mathrm{e}}$ s. avant J.-C., la prêtrise liée à un sanctuaire d'Asclépios est accordée au fils du fondateur et à ses descendants. Le règlement stipule qu'il s'agit de la prêtrise d'Asclépios et «des autres dieux installés » dans l'Asclépieion 24. L'expression ne permet pas de savoir s'il s'agit de statues ou d'autels, mais une chose est certaine : le document associe explicitement le dieu "titulaire" du lieu et les divinités qui partagent avec lui les honneurs des fidèles. C'est cela qu'évoque l' "installation" d'un dieu, que ce soit sous l'angle poétique ou sous la sécheresse d'un règlement civique.

Oreste ne précise évidemment pas ce qu'il fera en terre athénienne pour installer Artémis, mais le dossier "des marmites" laisse entendre qu'une offrande, sous la forme d'une bouillie ou de l'immolation d'un animal, constitue le contenu concret de l'hidrusis. On aurait donc affaire à ce que l'on appelle communément dans la littérature secondaire un « sacrifice de fondation ». La forme n'en semble pas codifiée une fois pour toutes selon des modalités techniques précises, mais relève, comme toute procédure sacrificielle en Grèce, du contexte de l'offrande (e.g. sphère domestique, sphère officielle), et des variations locales dans la manière de faire. Mais quel sens donner à une telle "fondation" ? S'agit-il vraiment d'une "consécration" et, dans l'affirmative, que recouvre ce terme en contraste avec, par exemple, la consécration d'une dédicace par déposition?

Je voudrais prendre un peu de recul par rapport à ce dossier en le mettant en perspective avec quelques traits particuliers de la représentation grecque du divin. Bon nombre de récits mettent en évidence le rapport des dieux aux cités des hommes et leur souci de recevoir la somme des honneurs qui leur sont dus ${ }^{25}$. La part d'honneur de chaque dieu, dont tout le mythe de souveraineté hésiodique précise les modalités d'attribution, ne trouve en fait sa pleine application que dans les cultes des cités. Ce sont des scénarios semblables que déploient les récits locaux sur la dévolution de la qualité de poliade à tel ou tel dieu. Le cas le plus connu est celui de la querelle entre Athéna et Poséidon à Athènes ${ }^{26}$, mais ce n'est pas le seul ${ }^{27}$. Dans sa critique acerbe d'une telle représentation des dieux, Lucien décrit adéquatement le processus du point de vue humain : les hommes se répartissent les dieux, les honorent et les revendiquent comme leurs concitoyens ${ }^{28}$. Le vocabulaire utilisé par les Grecs pour énoncer de telles procédures est particulièrement significatif : que ce soit un sanctuaire, un autel ou une statue, il s'agit, au sens strict, "d'installer le dieu » parmi les hommes. Il s'agit d'intégrer le dieu à la cité ou au groupe, de créer les conditions de la manifestation de sa divinité, de sa bienveillance au cœur même de l'espace de vie de ceux qui l'honorent. Sans minimiser la nécessité d'une sacralisation de l'espace prévu à cet effet, que le nom même d'hieron souligne à suffisance, le choix du registre sémantique de la "fondation" et de l' "installation" n'est pas innocent, même si, au cours du temps, ce type d'expression a pu se "démonétiser" et se figer.

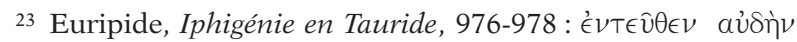

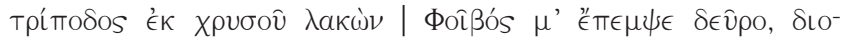

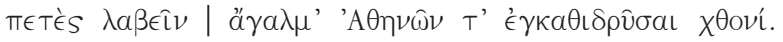

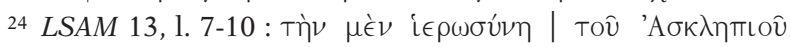

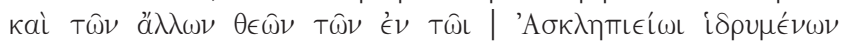

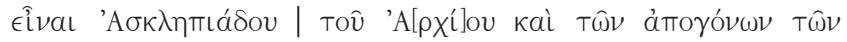

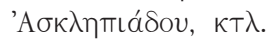

25 E.g. Rudhardt 1981, p. 227-244.

${ }^{26}$ Cf. Euripide, Phéniciennes, 854 ; Hérodote, VIII, 55 ; Pausanias, I, 26, 5.

27 Athéna et Poséidon à Trézène (Pausanias, II, 30, 6) ; Héra et Poséidon à Argos (Pausanias, II, 15, 5).

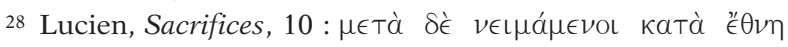

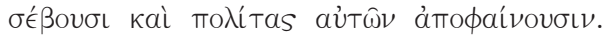




\section{"Installer un dieu"}

Comment, dès lors, "installer" mieux un dieu qu'en jouant une première fois la scène de l'hommage ? Comment mieux faire qu'en ouvrant la séquence de tous les hommages à venir ? Une inscription vient conforter une telle approche. Elle est très connue : il s'agit des deux parties du décret réglant les modalités de l'installation du xoanon d'Artémis Leucophryéné à Magnésie du Méandre à la charnière des $\mathrm{III}^{\mathrm{e}}$ et $\mathrm{II}^{\mathrm{e}}$ siècles avant J.-C. L' incipit de la première inscription résume en quelque sorte le contenu total du décret comme suit : "Sous la stéphanéphorie de Polukleidès fils de Pythodélès, au mois d'Agneon, à propos de l'installation du xoanon d'Artémis Leukophryéné dans le Parthénon maintenant construit pour elle et à propos de l'accomplissement en son honneur, chaque année le 6 du mois Artémision, de libations et de sacrifices, de la célébration conjointe par chacun des habitants de sacrifices devant les portes, selon les moyens de la maisonnée, sur les autels qui seront construits par eux à cet effet ${ }^{29}$. Dans le détail des données qui viennent ensuite, l'arrière-plan des hommages traditionnels aux dieux de la cité est clairement énoncé, ainsi que le lien particulier de la cité à son archègetis Artémis. Dès lors, au jour dit, le néocore et la prêtresse doivent accomplir le transfert de la déesse dans le Parthénon " avec un sacrifice le plus remarquable possible»; ce jour sera proclamé sacré à jamais et recevra le nom de Isitèria. Suivent alors les modalités de la célébration, procession, chœurs de jeunes filles, etc. Le nom du jour anniversaire de la kathidrusis pourrait être traduit par "Journée des célébrations inaugurales » ${ }^{30}$. Il n'est donc nullement question dans ce décret d'une procédure particulière visant à consacrer l'édifice restauré ou la statue ; il s'agit d'honorer la déesse avec le plus de faste possible, depuis le grand sacrifice accompli par la prêtresse jusqu'aux sacrifices des particuliers, modulés selon les moyens de chacun. La première célébration du 6 Artemision "installe" la statue en assurant la déesse qu'elle représente de la dévotion des habitants de Magnésie, en "captant" sa bienveillance et en lui donnant des gages pour l'avenir.

La "consécration" des statues divines s'inscrit dans cet ensemble particulier qu'est l'installation d'un dieu dans les cités des hommes. L'application du lexique de la fondation à la fois aux statues et aux autels montre à suffisance qu'il n'existe aucune opération spécifique autour de la statue, qui viserait à en faire "le dieu" ou "la déesse". Tant la statue que l'autel sont des lieux privilégiés où le contact peut s'établir avec un des représentants du monde supra-humain ${ }^{31}$. Et le sacrifice est l'instrument de ce contact.

Il reste un texte important à produire, pour tenter d'élucider davantage ce qu'il faut entendre par « lieu privilégié de la manifestation du divin ». Dans le Banquet des Sophistes d'Athénée, un grand nombre de pièces de vaisselle servant au banquet sont passées en revue, avec leur définition. Sous l'entrée kadiskos, Athénée précise qu'il s'agit d'« un récipient dans lequel sont établis les Zeus Ktesioi ». Il produit ensuite un extrait d'Antikleidès, auteur d'un Exègètikon de la période hellénistique : " Les sèmeia de Zeus Ktesios doivent être établis de cette façon : soit un kadiskos neuf à deux anses fermant avec un couvercle, il faut en couronner les anses avec de la laine blanche et, de son épaule droite et de son front (...) d'un fil de laine, y déposer ce qu'on trouve et y verser de l'ambroisie. L'ambroisie est faite d'eau pure, d'huile et d'un mélange de fruits divers ; tout cela mets-le dedans $» 32$.

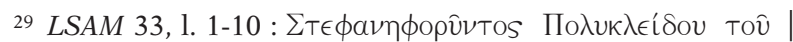

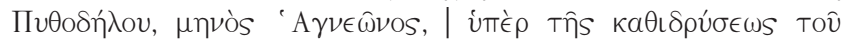

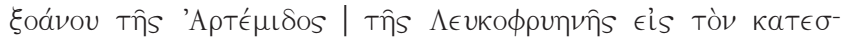

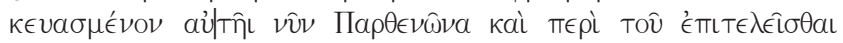

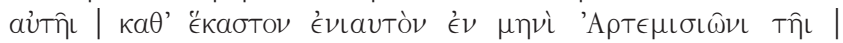

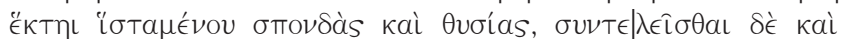

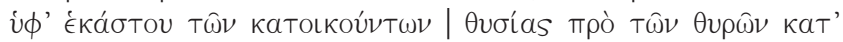

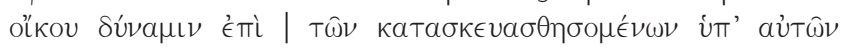
$\beta \omega \mu \omega \hat{\nu}$.

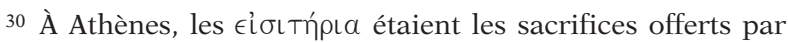
les magistrats avant d'entrer en charge ou en début d'année :

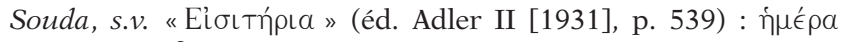

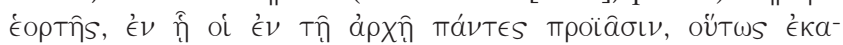

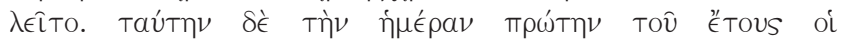

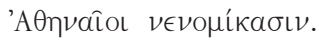

31 Par d'autres voies, je me rapproche du modèle interprétatif proposé par Scheer 2000, p. 35-43, 120-123, 143-146, 305 e.g., qui substitue à la dichotomie " statue = dieu » / " statue $\neq$ dieu » la vision d'une présence divine qui ne serait pas nécessairement stable et constante dans son rapport à la statue. Mais elle minimise le registre de l'installation (p. 114-115).

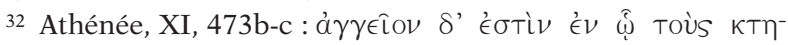

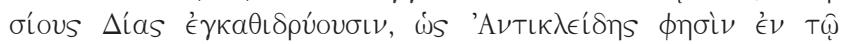

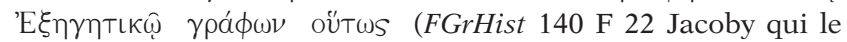
juge douteux sans argument, et reprend sous le nom d'Autokleidès le même fragment sous la référence $353 \mathrm{~F} 1$, à la suite d'une

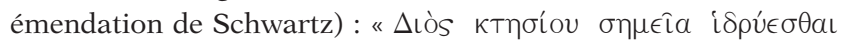


Ce texte prescriptif ${ }^{33}$ est complexe et son caractère lacunaire n'en facilite pas l'interprétation. Pour le présent propos, le terme de sèmeion est central. Que sont les sèmeia de Zeus Ktesios ? Si l'on s'en tient à la lettre du texte d'Antikleidès, ce sont les ingrédients qui composent la mixture versée dans le kadiskos ${ }^{34}$. Toutefois, le caractère fragmentaire de la prescription rituelle extraite par Athénée de l'Exègètikon peut avoir laissé dans l'ombre l'autel ou l'effigie du dieu (les sèmeia) que "fondait" la manipulation rituelle du kadiskos. Le dossier " des marmites" montre que l'offrande accompagnait l'établissement d'un autel ou d'une statue. En fait, kadiskos et "marmites" témoignent probablement de procédures analogues dans le cadre plus large des pratiques "fondatrices" dont nous n'avons plus trace.

Quoi qu'il en soit, ce passage d'Athénée rassemble plusieurs informations touchant à notre propos : tout d'abord, il y a une sorte d'équivalence entre le dieu et ses sèmeia quand on passe d'Athénée à Antikleidès ; ensuite, la "recette" de l'offrande non sanglante cherche manifestement à créer un effet de miroir entre le dieu et les ingrédients : l'ambroisie était réputée pour être la nourriture des dieux. Le "signe" de Zeus parmi les humains est donc installé selon une procédure qui joue à la fois sur la notion des honneurs à rendre au dieu et sur la reconnaissance de son statut divin. Mais le "signe" - qu'il soit le kadiskos lui-même avec son contenu, un autel ou une statue - est aussi une trace de la présence du dieu et le témoignage de la bienveillance que le fidèle escompte au fil de la répétition des offrandes.

Les signes en question servent à identifier le destinataire divin. Dans le cas des statues anthropomorphes, il s'agit essentiellement des attributs spécifiques de la divinité représentée et aussi - mais ce n'est malheureusement qu'une hypothèse - l'énonciation de son identité dans la prière qui devait accompagner le sacrifice ${ }^{35}$. Dans le cas des autels, le nom de la divinité au datif ou au génitif, inscrit sur le monument, peut être le "signe" de l'installation du dieu ${ }^{36}$. Ainsi, à Magnésie du Ménandre, l'inscription déjà évoquée stipule que les autels devant les maisons particulières seront recouverts d'une couche de stuc dans laquelle sera inscrite

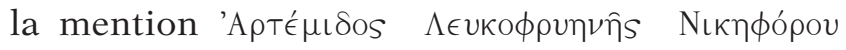
(1. 88-89). Un autre cas célèbre de l'élévation de ce type d'autel, bien étudié par Louis Robert, concerne la dévotion des particuliers à l'égard de la reine Arsinoé Philadelphe assimilée à Aphrodite. Dans ce cas précis, c'est du sable qui devra recouvrir les autels, attestant, selon Robert, l'assimilation de la reine à la déesse dans ses fonctions marines.

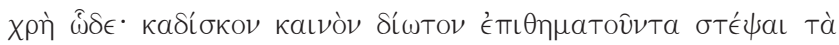

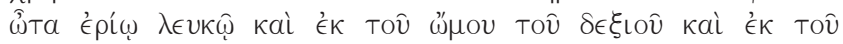

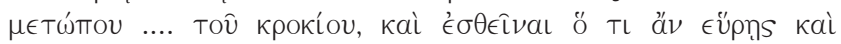

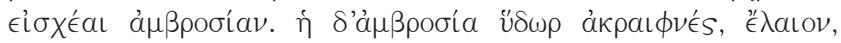

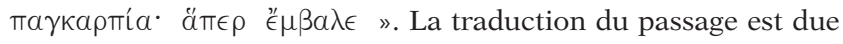
à Dominique Jaillard que je remercie de m'avoir transmis le manuscrit de son étude sur le Zeus Ktesios. L'article est aujourd'hui sorti de presse : cf. Jaillard 2004, p. 871-893, spéc. p. 873874 pour le texte, la traduction du passage d'Athénée et la mise au point sur Antikleidès/Autokleidès. À sa bibliographie, on peut maintenant ajouter R. Parker, Polytheism and Society in Athens, Oxford, 2005, p. 15-16, et P. Brulé, " La cité est la somme des maisons ». Un commentaire religieux, in : V. Dasen, M. Piérart

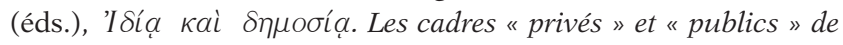
la religion grecque antique, Liège, 2005 (Kernos, suppl. 15), p. 27 53 , spéc. p. $29-48$.

33 La forme de cette prescription rituelle ressemble à s'y méprendre à celle des recettes médicales qui requièrent elles aussi des récipients neufs ou remis à neuf pour procéder aux décoctions ou aux mélanges : cf. Oribase, Collectiones Medicae VIII, 46, 16 (éd. Raeder I [1928], p. 297). Ce sont également des récipients neufs dont l'usage est recommandé pour un mélange à base de miel destiné aux Tritopatores "purs " dans la Loi sacrée de Sélinonte, A 15 (E. Lupu, Greek Sacred Law. A Collection of New Documents, Leiden, 2005, $\mathrm{n}^{\circ} 27$ ).

34 C'est l'interprétation de Jaillard 2004, p. 881, qui voit dans les sèmeia du dieu "l'objet composite que le rite "fabrique" ou "construit" ». Cet objet " présentifiera » désormais le dieu au milieu des autres jarres du tamieion. Le rite consiste à incorporer dans le kadiskos les matières sur la conservation desquelles le dieu veille (p. 886). Le sèmeion n'est « ni image ni signe arbitraire » (p. 882).

35 On dispose de quelques rares éléments à ce sujet pour la

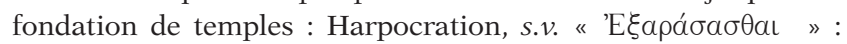

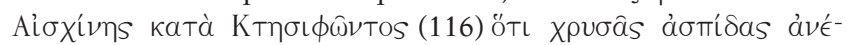

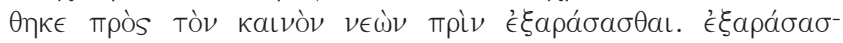

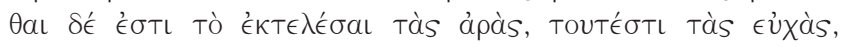

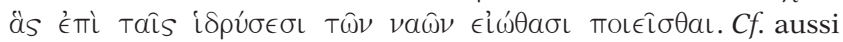
le type de discours dit « de consécration » chez Sopater (IV ${ }^{\mathrm{e}} \mathrm{s}$. ap. J.-C.), Scholia ad Hermogenis status seu artem rhetoricam II 5, 14

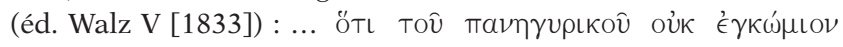

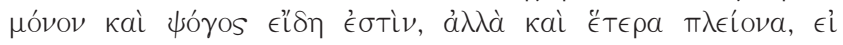

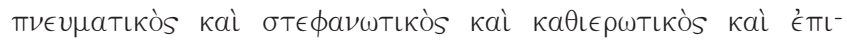

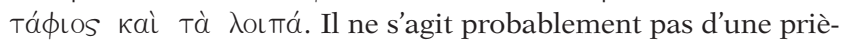
re à proprement parler.

36 L'équivalence "structurelle" entre nom et statue est poétiquement soulignée par Démocrite (68 F 142 [éd. Diels-Kranz ${ }^{6}$ )

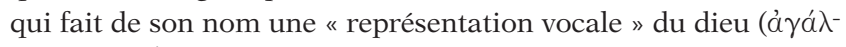

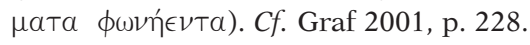


Le sable semble bien, en la circonstance, être une sorte de sèmeion de la déesse, son "signe" d'identification $^{37}$.

Par analogie, rappelons les manifestations divines respectives de Poséidon et d'Athéna lors de la querelle pour Athènes : la mer miniature et l'olivier témoignent de la bienveillance des deux divinités envers la cité, ils sont les "signes" de l'attention qu'ils comptent lui réserver. Mais ce type d'épiphanie est une évidence à laquelle tous les cultes ne peuvent pas prétendre. D’où la nécessité pour les humains d' "installer" eux-mêmes des preuves de cette bienveillance divine dans leur lieu d'existence, selon des procédures qui constituent autant de variations sur le thème de l'offrande sacrificielle. Une anecdote rapportée par Pausanias illustre d'une autre manière cette interprétation : au moment d'achever la statue du Zeus d'Olympie, Phidias aurait prié le dieu de lui signifier si le travail était à son goût; la foudre aurait aussitôt creusé un trou dans le sol «à l'endroit où ", écrit Pausanias, "il y avait encore de mon temps une hydrie pour ornement, celle de bronze $^{38}$ ». Il s'agit certainement d'une interprétation en termes "épiphaniques" de la cérémonie d'installation de la statue, peut-être liée à l'hydrie qui n'a aucun sens hors d'une telle référence ${ }^{39}$.

\section{Conclusion et perspectives...}

La première question posée dans l'introduction touchait à la distinction entre statue divine et statue "de culte". La distinction n'est pas aussi impertinente que d'aucuns l'ont récemment prétendu. Si l'on accepte la présente analyse sur l' "installation" d'une statue divine, c'est cette opération rituelle qui fait la différence entre la statue divine comme anathèma, - ce qui signifie simplement la propriété divine, - et l'agalma "fondé" comme sèmeion du dieu.

La seconde question touchait à la notion de "consécration". Elle semble trop vague pour être véritablement opérante en matière de statue de culte. Comme l'a constaté Fritz Graf, il n'est pas d'opération qui confère un statut véritablement divin à l'objet. Cependant, sa déposition dans le sanctuaire lui fait subir, en première instance, un transfert dans la propriété divine, qui est à proprement parler, en grec, une "consécration". L'objet est un anathèma. Ensuite, l' "installation ", l'hidrusis, consiste en une activation des "signes" de la bienveillance divine, par la mise en place des marques d'honneur requises par le dieu. Mais aucun terme grec n'est habilité à saisir une fois pour toutes l'objet ainsi "fondé" : d'où l'impossibilité de retrouver la "statue de culte" dans le lexique.

Une telle interprétation est également applicable à une notion qui a fait couler beaucoup d'encre. Il s'agit du terme ả $\dot{́} \delta \rho v \mu \alpha$. On y retrouve la racine hidr- à la base de notre réflexion. Le terme, relativement tardif, sert à désigner, surtout chez Strabon, un sanctuaire considéré comme la "filiale" d'un prestigieux modèle ${ }^{40}$. Mais la traduction par "filiale" ne fonctionne pas toujours. Dans certains de ces cas, aphidruma désigne clairement un modèle réduit d'une statue imitant une statue de culte. C'est le sens que L. Robert privilégiait dans bon nombre d'inscriptions ${ }^{41}$. Toutefois, un passage de Strabon reste problématique dans l'une et l'autre interprétation. Parlant de la fondation de Marseille, il raconte les épisodes merveilleux du transfert de culte de l'Artémis d'Éphèse par les Phocéens. Aristarché, noble femme d'Éphèse, est poussée par un rêve à se joindre aux colons, emportant avec elle, écrit

37 L. Robert, Sur un décret d'Ilion et sur un papyrus concernant des cultes royaux, in : Opera Minora Selecta, VII, 1990 [1966], p. 599-635, spéc. p. 616-634. Cf. la formulation de Vernant 1996, p. 367 : «La fonction de ce genre de sacra consiste à attester et à transmettre les pouvoirs que la divinité accorde en privilège à ses élus, plutôt qu'à faire connaître une "forme " divine au public. Le symbole ne représente pas le dieu, abstraitement conçu en lui-même et pour lui-même ; il ne cherche pas à instruire sur sa nature. Il exprime la puissance divine en tant que maniée et utilisée par certains individus, comme instrument de prestige social, moyen de prise et d'action sur autrui ». La dernière phrase ne s'adapte toutefois pas textuellement à la "statue de culte".

38 Pausanias, V, 11, 9 (trad. J. Pouilloux).

39 Sur l'impact de la statue sur les fidèles, voir le Discours olympique (XII) de Dion Chrysostome, de même que les descriptions de Pausanias (supra, note 38) et de Strabon (VIII, 3, 30 [C353-354]).

40 Strabon, VI, 2, 5 (C272) ; III, 5, 6 (C172) ; V, 3, 12 (C239), etc.

${ }^{41}$ L. Robert, Statues divines, in : Hellenica XIII, Paris, 1965, p. 120-125, dans une critique acerbe de J. Brunel, À propos des transferts de culte : un sens méconnu du mot ả Philologie, 27, 1953, p. 21-33. 


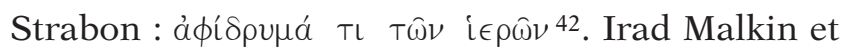
Claude Rolley refusent tous deux l'identification précise en «statue » ou en " modèle réduit de sanctuaire » pour conserver l'indétermination de l'expression " ce qui sert à fonder un culte parmi les objets sacrés ${ }^{43}$. Une fois l'indétermination du terme acceptée, c'est la notion de "fondation du culte" qui devient essentielle.

L'ensemble de la réflexion autour de l'installation d'un autel ou d'une statue vient appuyer encore davantage une telle interprétation : l'indétermination du terme aphidruma est liée à la variété des procédures employées pour "installer" une divinité, c'est-à-dire créer les conditions des honneurs à lui rendre, et pour déterminer un lieu privilégié de

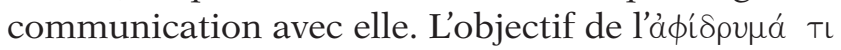
$\tau \omega \hat{\omega} \nu$ i $€ \omega \hat{\nu} \nu$ de Strabon devait être de favoriser dans le nouvel établissement la communication qui avait fait ses preuves à Éphèse et permettre de poursuivre l'échange "part d'honneur / bienveillance divine" entre le niveau humain et le niveau supra-humain. L'aphidruma peut être une image divine, mais ce n'est pas forcément le cas. Cette image n'est qu'une modalité parmi d'autres de la possibilité de présentifier un dieu. La logique qui est à l'œuvre n'est pas figurative dès qu'il s'agit de divin : elle est rituelle ${ }^{44}$.

Je me suis volontairement cantonnée au dossier littéraire et épigraphique du problème. Sans nul doute, l'archéologie a beaucoup à nous apprendre sur de telles pratiques puisque les instruments de cette "installation" pouvaient rester en place. Encore faut-il bien identifier de tels dépôts, en distinguant ce que les germanophones appellent Bauopfer, c'est-à-dire les dépositions liées d'une manière ou d'une autre à la stabilisation de l'édifice sacré, et les procédures d'installation analysées ici. L'idéal serait de parvenir à en isoler les composantes et à remettre le tout en perspective, en dépassant la simple étiquette de "consécration".

42 Strabon, IV 1, 4 (C179). Sur le contexte de l'oracle parallèle au rêve d'Aristarché, $c f$. F. Salviat, La source ionienne : Apatouria, Apollon Delphinios et l'oracle, l'Arstarcheion, in : A. Hermary, H. Tréziny (éds.), Études massaliètes 6. Les Cultes des cités phocéennes. Actes du Colloque International Aix-enProvence / Marseille 4-5 juin 1999, Aix-en-Provence, 2000, p. $25-$ 31, spéc. p. 30-31.

43 I. Malkin, What is an aphidruma ?, Classical Antiquity, 10, 1991, p. 77-96, spéc. p. 78-87. Cf. le résumé du dossier chez Bettinetti 2001, p. 54-63, auquel il faut ajouter Cl. Rolley, Encore

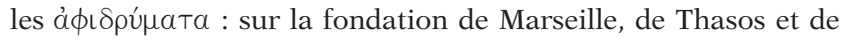
Rome, AION(ArchStAnt), n.s. 4, 1997, p. 35-43, spéc. p. 37-43. NB. Depuis le dépôt de cet article ont paru deux notes sur la notion, qui font écho à l'analyse ici proposée : A. Anguissola, Note on Aphidruma 1: Statues and their function; 2: Strabo on the transfer of cults, Classical Quarterly, 56, 2006, p. 641-646.

${ }^{44}$ Cf. D. Jaillard, Le pilier hermaïque dans l'espace sacrificiel, MEFRA, 113, 2001, p. 341-363.

\section{Abréviations bibliographiques}

Bettinetti 2001 : S. Bettinetti, La statua di culto nella pratica rituale greca, Bari, 2001.

Graf 2001 : Fr. Graf, Der Eigensinn der Götterbilder in antiken religiösen Diskursen, in : G. Boehm (éd.), Homo Pictor, Leipzig, 2001 (Colloquium Rauricum, Band 7), p. 227-243.

Jaillard 2004 : D. Jaillard, « Images » des dieux et pratiques rituelles dans les maisons grecques. L'exemple de Zeus Ktesios, MEFRA, 116, 2004, p. 871-893.

Linant de Bellefonds 2004 : P. Linant de Bellefonds et al., Cult images/Images de culte/Kultbilder/Immagini di culto, in : ThesCRA (Thesaurus Cultus et Rituum Antiquorum) II, Los Angeles, 2004, p. 417-507.

Rudhardt 1981 : J. Rudhardt, Du mythe, de la religion grecque et de la compréhension d'autrui, Genève, 1981.

Scheer 2000 : T. Scheer, Die Gottheit und ihr Bild. Untersuchungen zur Funktion griechischer Kultbilder in Religion und Politik, Munich, 2000 (Zetemata, 105).

Vernant 1996 : J.-P. Vernant, De la présentification de l'invisible à l'imitation de l'apparence, in : Id., Entre mythe et politique, Paris, 1996, p. 359-377 [reprise d'un article publié en 1983]. 